\title{
Numerical Analysis of the Resin Transfer Molding Process via PAM- RTM Software
}

\author{
I.R. Oliveira ${ }^{1, a}$, S.C Amico ${ }^{2, b}$, J.A. Souza ${ }^{3, c}$ and A.G. Barbosa de Lima ${ }^{1, d}$ \\ ${ }^{1}$ Department of Mechanical Engineering, Federal University of Campina Grande (UFCG), Zip Code \\ 58429-900, Campina Grande-PB, Brazil. \\ ${ }^{2}$ Department of Materials Engineering, Federal University of Rio Grande do Sul (UFRGS), Zip \\ Code 91501-970, Porto Alegre-RS, Brazil. \\ ${ }^{3}$ Department of Mechanical Engineering, Federal University of Rio Grande (FURG), Zip Code \\ 96201-900, Rio Grande-RS, Brazil.
}

arodrigues.iran@hotmail.com, bamico@ufrgs.br, cjasouza@furg.br, 'dgilson@dem.ufcg.edu.br

Keywords: RTM, flow simulation, linear injection, pressure

\begin{abstract}
This work aims to investigate the infiltration of a $\mathrm{CaCO}_{3}$ filled resin in fibrous porous media (resin transfer molding process) using the PAM-RTM software. A preform of glass fiber mat (fraction 30\%), with dimensions $320 \times 150 \times 3.6 \mathrm{~mm}$, has been used in rectilinear injection experiments conducted at room temperature and injection pressure $0.25,0.50$ and 0.75 bar. The polyester resin contain $0 \%$ and $40 \% \mathrm{CaCO}_{3}$. The numerical results were evaluated by direct comparison with experimental data. The flat flow-front profile of the rectilinear flow was reached approximately half length of the mold. It was observed, that the both velocity infiltration and permeability have decreased with increasing the $\mathrm{CaCO}_{3}$ content, thus, increasing the time to processing of the composite material.
\end{abstract}

\section{Introduction}

Resin transfer moulding (RTM) increasingly becomes an important processing technology which has found wide applications to fabricate net-shape polymer-matrix composites, ranging from nonstructural components with simple shapes, to structural parts with complex geometries. The RTM process provides various advantages in producing high-performance composites such as rapid processing with low labour requirement, potential to prevent the emission of hazardous volatile materials, e.g., styrene, and versatile design of fibrous reinforcements. These capabilities make the process an attractive technique to be used in various industrial segments such as automotive, transportation and aerospace [1]. For a good RTM molding becomes is necessary to know and control different process parameters such as fluid viscosity, injection pressure, volumetric fraction of fiber, temperature and permeability of the medium. The injection pressure and temperature are the main factors, so, the optimized use of these parameters are desirable for a correct filling of the mold, this increasing productivity [2].

In addition to experimental studies, numerical analysis software have been frequently used to predict the resin behavior within the preform/mold along the RTM process, evaluate the filling time (which should be less than the resin's gel time), evaluate deficient impregnation points and adequately to determine the resin injection points and vent ports [3]. For this purpose commercial packages were developed, such as PAM-RTM or RTM-Worx, where numerical models were codefied in order to obtain a prediction of the resin flow [4].

PAM-RTM Software is a commercial software, developed by ESI-Group, that includes many packages covering almost all LCM technologies. The RTM module can work with a full 3D model and calculates the resin flow using the resin viscosity, the permeability of the porous media, and appropriates boundary conditions imposed, which can include pressure or flow-rate inlets, vents and eventually temperature (for this last variation a different module called Heated-RTM is available). The calculations of the flow are based on Darcy's law and the continuity equation, which has been widely used by researchers to describe the flow mechanisms [5]. 
In complement for these cited studies, this work aims to simulate a resin rectilinear infiltration in a glass fiber mat fibrous media using the PAM-RTM software, and evaluate the influence of process parameters, such as resin viscosity, porous media permeability and $\mathrm{CaCO}_{3}$ content, at the resin infiltration velocity in the porous media.

\section{Mathematical model}

In this work we use a mold with dimensions $320 \times 150 \times 3.6 \mathrm{~mm}$ (Figure 1). In the mold, the rectangular region is specially designed to facilitate the formation of the linear profile of the resin forward advancement. This mold outline is required to be able, in laboratory experiments, to determinate the porous media permeability from the experimental data.

In all simulations it was assumed an isothermic and incompressible Newtonian fluid flow through porous medium. The mathematical model used is based on the Darcy`s law and continuity equation, and it is given as follows $[3,7]$.

$$
\begin{gathered}
\vec{v}=-\frac{1}{\mu} k \nabla P \\
\nabla \cdot \vec{v}=0
\end{gathered}
$$

where $\vec{v}$ is the fluid velocity vector $[\mathrm{m} / \mathrm{s}] ; k$ is the porous media permeability $\left[\mathrm{m}^{2}\right] ; \nabla \mathrm{P}$ is the pressure gradient $[\mathrm{Pa} / \mathrm{m}]$ and $\mu$ is the resin viscosity $[\mathrm{Pa} . \mathrm{s}]$.

The model was solved using a non-conforming finite element approximation. The mesh obtained by PAM-RTM commercial code has 7077 elements and 3685 nodes, with better refinement in the zone near to the injection region. The pressure is discontinuous along the inter-element boundaries except at the middle nodes, and filling factors are associated with the mesh elements [9]. Table 1 summarizes the initial and boundary conditions for the studied cases.

\begin{tabular}{|c|c|c|c|}
\hline \multicolumn{2}{|c|}{ Filled resin with $0 \% \mathrm{CaCO}_{3}$} & \multicolumn{2}{|c|}{ Filled resin with $40 \% \mathrm{CaCO}_{3}$} \\
\hline \multicolumn{2}{|c|}{$\begin{array}{l}\mathrm{P}_{\text {inlet }} \rightarrow \text { varying from } 0 \text { to } 22100 ; 0 \\
\text { to } 45900 \text { and } 0 \text { to } 70100 \\
\mathrm{~Pa} \text { (manometric) }\end{array}$} & \multicolumn{2}{|c|}{$\begin{array}{l}\mathrm{P}_{\text {inlet }} \rightarrow \text { varying from } 0 \text { to } 21200 ; 0 \\
\text { to } 45100 \text { and } 0 \text { to } 70100 \mathrm{~Pa} \\
\text { (manometric) }\end{array}$} \\
\hline \multicolumn{2}{|c|}{ Wall $\rightarrow$ zero pressure gradient } & \multicolumn{2}{|c|}{ Wall $\rightarrow$ zero pressure gradient } \\
\hline \multicolumn{2}{|c|}{$\mathrm{P}_{\text {front flow }}=0 \mathrm{~Pa}$ (manometric) } & \multicolumn{2}{|c|}{$\mathrm{P}_{\text {front flow }}=0 \mathrm{~Pa}$ (manometric) } \\
\hline \multicolumn{2}{|c|}{$\mathrm{T}_{\text {resin,inlet }}$} & \multicolumn{2}{|c|}{$\mathrm{T}_{\text {resin,inlet }}$} \\
\hline 0 to $22100 \mathrm{~Pa}$ & $292.35 \mathrm{~K}$ & 0 to $21200 \mathrm{~Pa}$ & $292.15 \mathrm{~K}$ \\
\hline 0 to $45900 \mathrm{~Pa}$ & $292.65 \mathrm{~K}$ & 0 to $45100 \mathrm{~Pa}$ & $291.95 \mathrm{~K}$ \\
\hline 0 to $70100 \mathrm{~Pa}$ & $291.85 \mathrm{~K}$ & 0 to $70100 \mathrm{~Pa}$ & $293.25 \mathrm{~K}$ \\
\hline
\end{tabular}

Table 1 - Initial and boundary conditions for the studied cases.

For the numerical results validation, experiments were performed injecting orthophthalic polyester resin in fiberglass mat for two different infiltration cases: a) Pure resin $\left(0 \% \mathrm{CaCO}_{3}\right)$, density $\rho=$ $1190 \mathrm{~kg} / \mathrm{m}^{3}$, fiber volume fraction $\mathrm{V}_{\mathrm{f}}=30 \%$, porosity $\varphi=0.7$, and b) Filled resin with $40 \% \mathrm{CaCO}_{3}$, composite density $\rho=1430 \mathrm{~kg} / \mathrm{m}^{3}$, fiber volume fraction $\mathrm{V}_{\mathrm{f}}=30 \%$, porosity $\varphi=0.7$ the calcium carbonate has commercial granulometry (as sold in the Brazilian market). Detail about the equipment and experimental procedure can be found in $[3,6,7,8]$. 


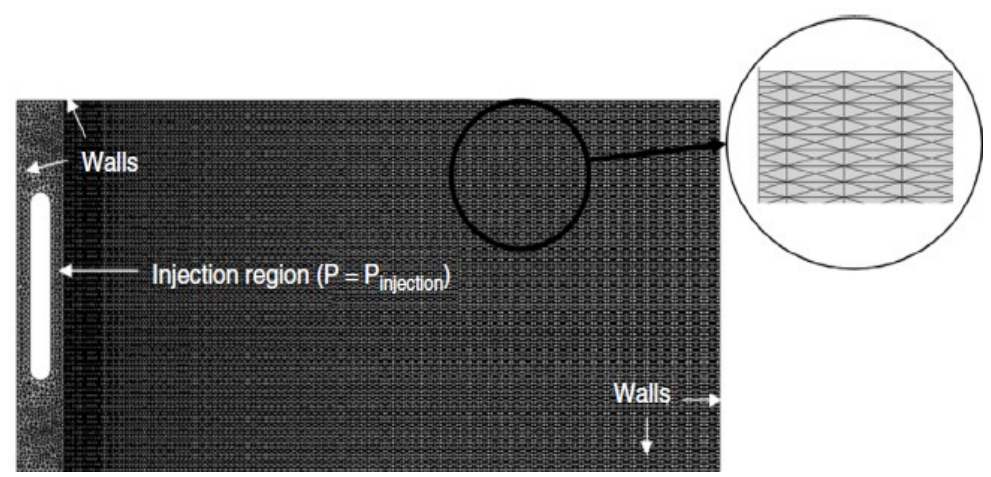

Figure 1 - 2D mesh used at the computational simulations with PAM-RTM.

\section{Results and Discusions}

Table 2 shows results for resin viscosity and porous media permeability as a function of calcium carbonate content and injection pressure. It is observed that the addition of $\mathrm{CaCO}_{3}$ affect slightly the permeability and increases the viscosity.

With the increasing in fluid viscosity, have an increases in the filling time (the time for completion the fibrous media with resin) as show in Table 3, where the experimental results of filling time and flow front position are compared with the PAM-RTM numerical solution. We notice that a good agreement was obtained in all simulations.

Table 2 - Resin viscosity and porous media permeability data for each experiment.

\begin{tabular}{cccc}
\hline & Viscosity $(\mathrm{cP})$ & Maximum injection pressure (bar) & Permeability $\left(\times 10^{-10} \mathrm{~m}^{2}\right)$ \\
\hline $0 \% \mathrm{CaCO}_{3}$ & 330 & 0.221 & 1.700 \\
$40 \% \mathrm{CaCO}_{3}$ & 2113 & 0.212 & 1.000 \\
\hline $0 \% \mathrm{CaCO}_{3}$ & 330 & 0.459 & 2.549 \\
$40 \% \mathrm{CaCO}_{3}$ & 2113 & 0.451 & 0.300 \\
\hline $0 \% \mathrm{CaCO}_{3}$ & 330 & 0.701 & 2.455 \\
$40 \% \mathrm{CaCO}_{3}$ & 2113 & 0.701 & 0.446 \\
\hline
\end{tabular}

Table 3 - Comparison between experimental and numerical results. Filling time (s) Position of flow front $(\mathrm{m})$

\begin{tabular}{ccccccc}
$\mathrm{CaCO}_{3}$ content (\%) & Experimental & Numerical & Error (\%) & Experimental & Numerical & Error (\%) \\
\hline 0 & 3120 & 3102 & 0.50 & 0.32 & 0.32 & 0.0 \\
40 & 4560 & 4507 & 1.00 & 0.11 & 0.12 & 8.3 \\
0 & 995 & 997 & 0.20 & 0.32 & 0.32 & 0.0 \\
40 & 6060 & 6151 & 1.40 & 0.08 & 0.08 & 0.0 \\
0 & 674 & 677 & 0.40 & 0.32 & 0.32 & 0.0 \\
40 & 3000 & 3026 & 0.85 & 0.11 & 0.11 & 0.0 \\
\hline
\end{tabular}

Figure 2 illustrates the pressure behavior within the preform at different elapsed times and calcium carbonate content in the resin. We can see that the higher pressure occurs on the injection port and lower pressure is verified in the vent port, as expected, because maximum and minimum pressures correspond to boundary conditions for the studied physical problem.

The flow front advance for both cases during the resin injection is shown in Figs.3-5. It is noticed that the results obtained with numerical solution had good agreement, with experimental data. The rectilinear flow front profile has occurred at approximately half length of the mold. Initially, at the region close to the injection port, the flow has 2D characteristics and the flow front assumes a ring (radial) shape form in the main flow direction. When the pressure gradient become linear, the flow front tends to become rectilinear (1D). 
From the analysis of the Fig.5 we can see that the PAM-RTM numerical solution is in good agreement with experimental results. A large error is observed in the nonlinear (close to the injection section) region. The difference is probably due to the $2 \mathrm{D}$ characteristic of the experimental setup on which the injection is performed through the bottom of the mold while in the numerical solution prescribed pressure is specified at the borders of the injection port. Besides, the permeability was determined based on the $1 \mathrm{D}$ rectilinear flow $(\mathrm{t}>100 \mathrm{~s})[3,6,7]$.

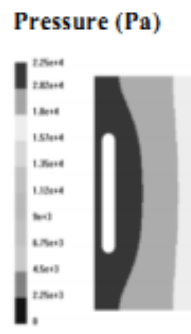

a) $\mathrm{t}=2773 \mathrm{~s}\left(0 \% \mathrm{CaCO}_{3}, \mathrm{P}=0.25\right.$ bar $)$

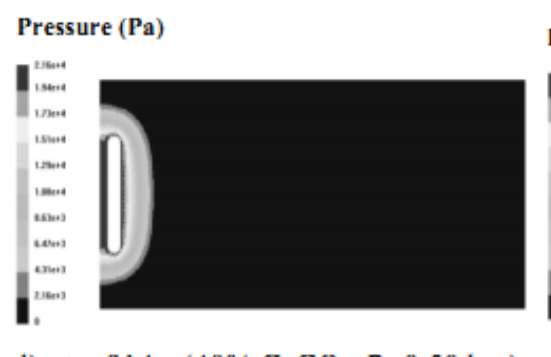

d) $\mathrm{t}=914 \mathrm{~s}\left(40 \% \mathrm{CaCO}_{3}, \mathrm{P}=0.50 \mathrm{bar}\right)$

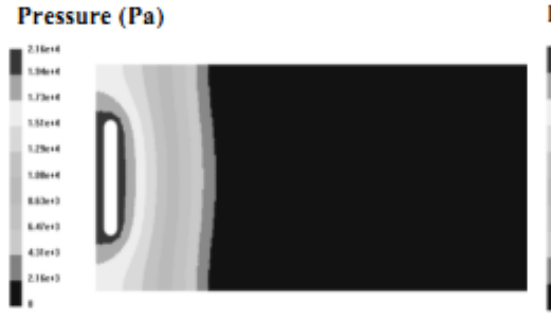

b) $\mathrm{t}=2799 \mathrm{~s}\left(40 \% \mathrm{CaCO}_{3}, \mathrm{P}=0.25\right.$ bar $)$ Pressure (Pa)

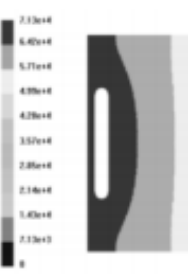

e) $\mathrm{t}=662 \mathrm{~s}\left(0 \% \mathrm{CaCO}_{3}, \mathrm{P}=0.75\right.$ bar $)$
Pressure $(\mathbf{P a})$

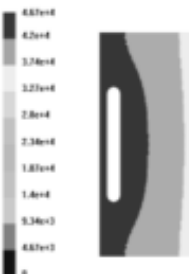

c) $\mathrm{t}=912 \mathrm{~s}\left(0 \% \mathrm{CaCO}_{3}, \mathrm{P}=0.50 \mathrm{bar}\right)$ Pressure (Pa)

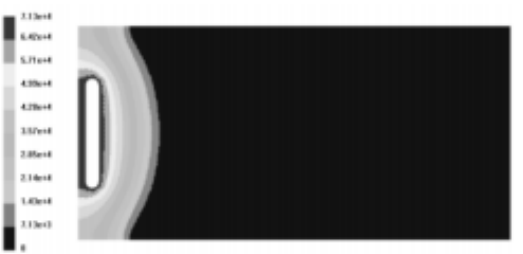

f) $\mathrm{t}=668 \mathrm{~s}\left(40 \% \mathrm{CaCO}_{3}, \mathrm{P}=0.75\right.$ bar $)$

Figure 2- Pressure field obtained with PAM-RTM software.

a)

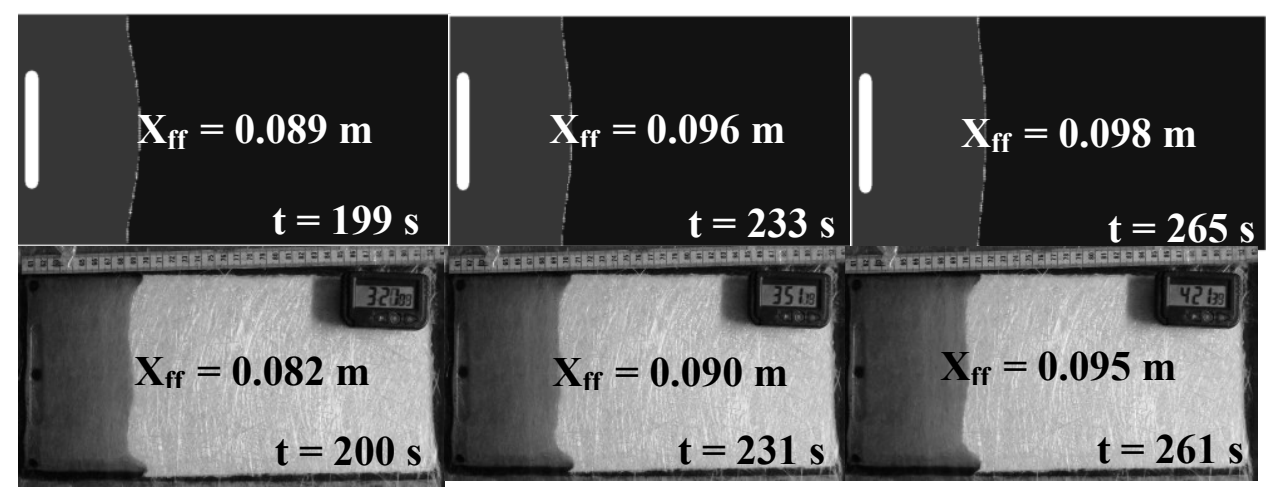

Figure 3 - a) Predicted (PAM-RTM) and b) Experimental front flow for the pure resin $\left(0 \% \mathrm{CaCO}_{3}\right.$ content, $\mathrm{P}=0.25$ bar) at different times.

a)

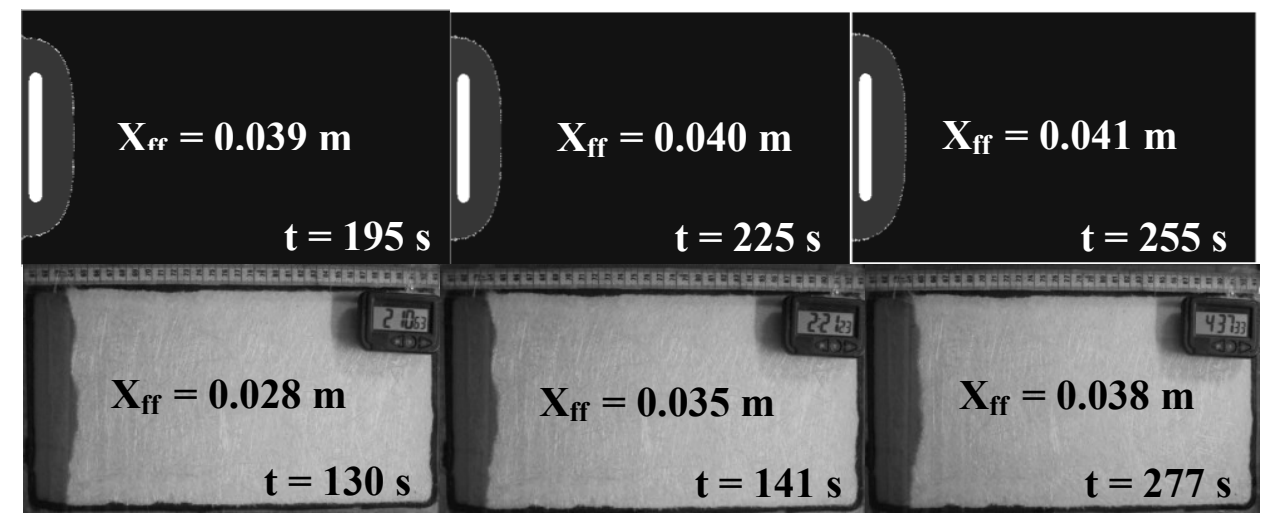

Figure 4 - a) Predicted (PAM-RTM) and b) Experimental front flow for the $\mathrm{CaCO}_{3}$ filled resin $\left(40 \% \mathrm{CaCO}_{3}\right.$ content, $\mathrm{P}=0.25$ bar) at different times. 


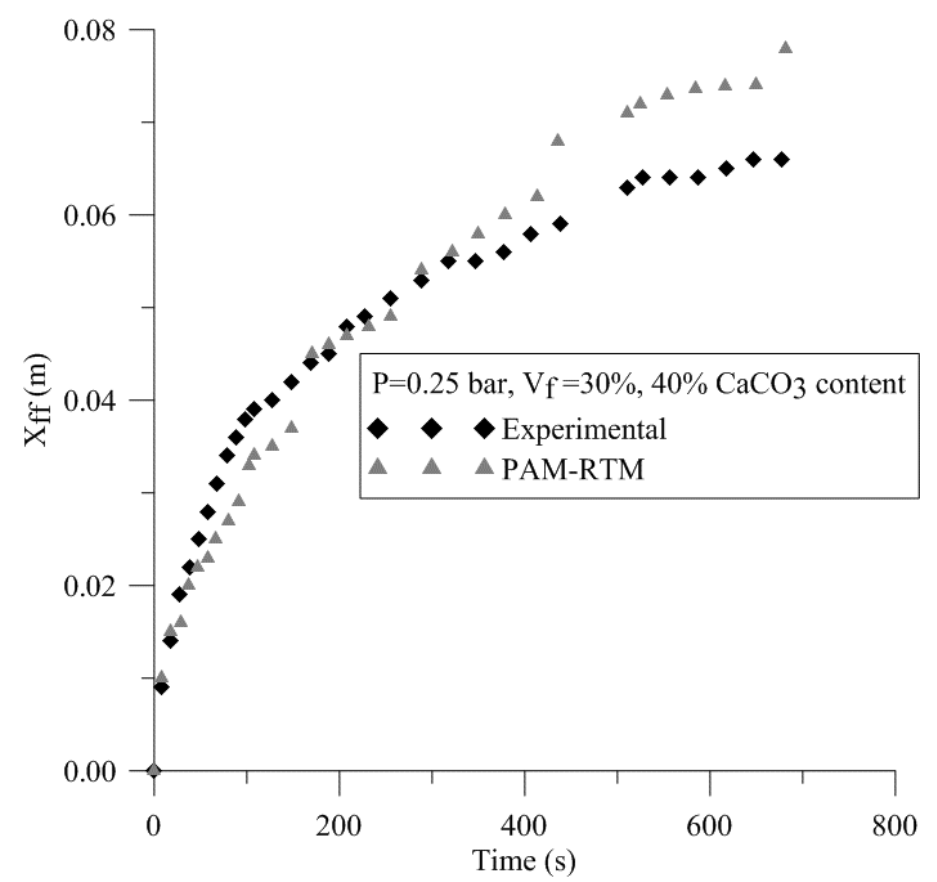

Figure 5 - Resin flow front position inside the mold versus time $\left(40 \% \mathrm{CaCO}_{3}\right.$ content, $\mathrm{P}=0.25$ bar).

\section{Conclusions}

This paper provides numerical and experimental information about RTM process. The PAM-RTM commercial software has been applied to simulate resin flow inside the porous fibrous. From the results presented we can be concluded that:

$>$ The computational solution represented well the physics involved in RTM process. It was possible to simulate the total resin filling time for both cases $\left(0\right.$ and $\left.40 \% \mathrm{CaCO}_{3}\right)$ and predict the fluid flow front profile into the mold.

$>$ Numerical results showed good agreement with the experimental data in terms of front flow position, filling time and injection pressure.

- Addition of $\mathrm{CaCO} 3$ in the resin modifies the porous media permeability values, increases viscosity and the filling time.

\section{Acknowledgements}

The authors thank to CNPq, FINEP and CAPES for financial support, and to the authors referred in this text that contributed for improvement of this work.

\section{References}

[1] A. Shojaei: Comput. Meth. Appl. Mech. Eng. Vol.3 (2006), n.9, p. 1434-1450.

[2] I. R. Oliveira, S.C. Amico, A. Souza, R. Barcella and A.G.B. Lima: Int. Jnl. of Multiphysic, Vol. 7 (2013), p. 125

[3] I. R. Oliveira, S. C. Amico, F. F. Luz, J. A. Souza, R. Barcella and A. G. B. Lima: Defect Diffusion Forum, Vol. 334-335 (2013).

[4] C. Demaria, E. Ruiz and F.Trochu: Polym. Compos, Vol. 28 (2007), p.812

[5] E. Poodts, G. Minak , L. Mazzocchetti and L. Giorgini: Compos. Part B, Vol.56 (2014), p.673

[6] J. C. Gelin, A. Cherouat, P. Boisse, and H. Sabhi: Comp. Sci. Technol. Vo. 56 (1996), p.711 
[7] C. D.Rudd, K. N. LAC and C. G. E. Mangin: Liquid Moulding, Structural Reaction Injection Moulding and Related Processing Techniques. Woodhead, Publishing Limited, 1997.

[8] F. F. Luz, S. C. Amico, J. A. Souza, E. S. Barbosa and A. G. B. Lima, in: Numerical Analysis of Heat and Mass Transfer in Porous Media, edited by J. M. P. Q. Delgado, Antonio Gilson Barbosa de Lima and Marta Vázquez da Silva, Springer, Heidelberg (2012).

[9] PAM-RTM - User`s Guide \& Tutorials, 2009. 